\title{
Long-term monitoring of intracranial pressure in normal pressure hydrocephalus and other CSF disorders
}

\author{
Zofia Czosnyka $^{1} \cdot$ Marek Czosnyka $^{1}$ (D)
}

Received: 16 July 2017 / Accepted: 19 July 2017 / Published online: 29 July 2017

(C) Springer-Verlag GmbH Austria 2017

\begin{abstract}
Long-term monitoring of intracranial pressure is a useful method in the diagnosis of complex hydrocephalus and CSF disorders. This editorial discusses various criteria for the interpretation of ICP monitoring in normal pressure hydrocephalus, idiopathic intracranial hypertension and in patients with implanted hydrocephalus shunts.
\end{abstract}

Keywords Intracranial pressure $\cdot$ Pressure waves $\cdot$ Normal pressure hydrocephalus $\cdot$ Idiopathic intracranial hypertension

Intracranial pressure (ICP) in normal pressure hydrocephalus (NPH) is a difficult subject because the pressure, as stated in the name of this disease, remains normal most of the time. Therefore, additional measures (secondary indices of decreased compensatory reserve, vasogeninic instability, waves of ICP, etc.) are usually taken into account. To complicate this subject, there are no known control-monitoring data in healthy subjects (with the exception of short-term infusion tests; a small cohort has been studied and published [1]).

The article printed in this issue by Chari and colleagues [3] gives solid answers to many questions concerning the interpretation of long-term intraparenchymal ICP monitoring. The authors are probably right that clinically useful information can be derived from the mean ICP and pulse amplitude of the ICP, with its relationship to body posture and age dependence.

Marek Czosnyka

mc141@medschl.cam.ac.uk

1 Brain Physics Laboratory, Division of Neurosurgery, Department of Clinical Neurosciences, Cambridge University Hospitals, Cambridge, UK
Additionally, they highlight the differences between different types of CSF disorders.

Intraparenchymal ICP monitoring is an invasive method. It cannot be blanket recommended in all cases. On the contrary, in most patients brain imaging, clinical investigation and neuropsychologic tests give a sufficient basis for a decision about treatment. However, in many complex cases, the longterm ICP monitoring or infusion test [10] appears to be indispensable. To remember how to interpret ICP monitoring properly, rehearsing some useful principles is worthwhile:

ICP is a multifactorial modality; generally it contains four distinct components: two vasogenic components, arterial and venous, a component related to the cerebrospinal fluid (CSF) circulation and the pressure-volume compensation, and a component secondary to volumetric changes of brain tissue. In acute states, all four components are important, and their knowledge is essential to determine the optimal ICP control strategy. In NPH, vasogenic and CSF circulatory components are dominant.

Vasogenic components can be monitored as specific ICP waves and transients. Regular waves are: a pulse wave (of fundamental frequency equal to a heart rate), respiratory wave (of fundamental frequency equal to a respiratory rate) and more or less regular slow vasogenic waves (of frequency between $0.005 \mathrm{~Hz}$ to $0.05 \mathrm{~Hz}$, commonly named 'B waves'). Vasogenic transients related to changes in arterial pressure, plateau waves (seldom in NPH and IIH), transients related to changes in body posture, head elevation or rotation, etc., are commonly observed.

Vasogenic components are almost always present; they show that overnight ICP recordings usually look quite irregular.

Classically 'B waves' recorded for more than $60 \%$ of the time in overnight monitoring of ICP have a positive predictive 
power for improvement after shunting in NPH [2]; however, there are serious studies challenging this opinion [9].

Increased pulse waveform (peak-to-peak magnitude $>5 \mathrm{mmHg}$ ) was also reported to correlate with good outcome after shunting in NPH [5]; however, other centers so far cannot confirm this. The shape of the pulse amplitude, particularly the increase of peak P2 over P1, is advocated as a signature of a diminished pressure-volume compensatory reserve; however, this finding has never been convincingly demonstrated as a predictor for improvement after surgery. It is not easy to assess the proportion between these peaks automatically; however, certain computer-assisted solutions exist [6].

We introduced an index called the RAP, the correlation coefficient between slow changes in the amplitude of the ICP and mean ICP. Value of RAP close to zero indicates a good compensatory reserve and a RAP $>0.6$ a poor compensatory reserve. Like with $\mathrm{P} 1 / \mathrm{P} 2$ peaks, we cannot show a convincing correlation between the RAP and outcome after shunting.

Our own 'Cambridge inventory' for the interpretation of overnight ICP monitoring can be summarized as follows:

We only interpret the ICP recording in the horizontal body position during physiologic sleep. If the averaged ICP overnight is $<12 \mathrm{mmHg}$, we interpret it as normal. From 12 to $15 \mathrm{mmHg}$, it is borderline, above $15 \mathrm{mmHg}$ elevated.

During slow vasogenic waves, the averaged level of ICP increases. If 10-s averages of the ICP increase during these waves above $25 \mathrm{mmHg}$, we interpret them as pathologic.

If the overall integrated magnitude of slow waves overnight exceeds $1.5 \mathrm{mmHg}$, we treat them as abnormal. If the overnight RAP index exceeds 0.6 , we interpret this as a signature of depleted compensatory reserve. Finally, when overnight the averaged peak-to-peak amplitude of the pulse ICP waveform exceeds $5 \mathrm{mmHg}$, we also interpret this finding as abnormal.

In IIH, the mainly vasogenic venous component of ICP is responsible for elevated ICP. It is commonly caused by stenosis in the pathway of cerebral venous outflow, which is reflected by the tight correlation between the sagittal sinus pressure and ICP (CSF pressure) [7]. The ICP is elevated above $20-25 \mathrm{mmHg}$, the pulse amplitude is in normal range (1-3 $\mathrm{mmHg}$ ), slow vasogenic waves are usually in normal range, and the compensatory reserve is depleted (RAP usually above 0.6 ).

The value of overnight ICP monitoring can be clearly appreciated in patients with an implanted shunt. In these cases, monitoring is performed to assess how optimally the shunt is working. When the overnight averaged ICP is in normal range (below the 'critical' threshold for an implanted shunt [4]), the pulse amplitude is in normal range $(1-3 \mathrm{mmHg})$, slow vasogenic waves are in normal range $(<1.5 \mathrm{mmHg})$, and the RAP is in normal range $(<0.5)$, there is no evidence of shunt malfunction. Otherwise, shunt underdrainage may be suspected. In cases of overdrainage, a comparison of the ICP in the horizontal and vertical positions [8] is useful. If the ICP decreases over a longer period (usually with the patient sitting up in bed) below $-10 \mathrm{mmHg}$, overdrainage can be expected.

In conclusion, if the clinical and radiologic pictures are clear, shunting surgery can be performed with confidence. If the overall picture is not clear, long-term monitoring or infusion tests are useful in making decisions about optimal management. Additionally, in this way the valuable baseline material can be recorded for comparison if any complications arise after shunting.

\section{References}

1. Albeck MJ, Børgesen SE, Gjerris F, Schmidt JF, Sørensen PS (1991) Intracranial pressure and cerebrospinal fluid outflow conductance in healthy subjects. J Neurosurg 74(4):597-600

2. Børgesen SE, Gjerris F (1982) The predictive value of conductance to outflow of CSF in normal pressure hydrocephalus. Brain 105(Pt 1):65-86

3. Chari A, Dasgupta D, Smedley A et al. (2017) Intraparenchymal intracranial pressure monitoring for hydrocephalus and cerebrospinal fluid disorders. Acta Neurochir. doi:10.1007/s00701-017-3281-2

4. Chari A, Czosnyka M, Richards HK, Pickard JD, Czosnyka ZH (2014) Hydrocephalus shunt technology: 20 years of experience from the Cambridge shunt evaluation laboratory. J Neurosurg 120(3):697-707

5. Eide PK, Brean A (2010) Cerebrospinal fluid pulse pressure amplitude during lumbar infusion in idiopathic normal pressure hydrocephalus can predict response to shunting. Cerebrospinal Fluid Res 12(7):5

6. Hu X, Hamilton R, Baldwin K, Vespa PM, Bergsneider M (2012) Automated extraction of decision rules for predicting lumbar drain outcome by analyzing overnight intracranial pressure. Acta Neurochir Suppl. 114:207-212

7. Pickard JD, Czosnyka Z, Czosnyka M, Owler B, Higgins JN (2008) Coupling of sagittal sinus pressure and cerebrospinal fluid pressure in idiopathic intracranial hypertension-a preliminary report. Acta Neurochir Suppl 102:283-285

8. Schuhmann MU, Sood S, McAllister JP, Jaeger M, Ham SD, Czosnyka Z, Czosnyka M (2008) Value of overnight monitoring of intracranial pressure in hydrocephalic children. Pediatr Neurosurg 44(4):269-279

9. Stephensen H, Andersson N, Eklund A, Malm J, Tisell M, Wikkelsö C (2005) Objective B wave analysis in 55 patients with non-communicating and communicating hydrocephalus. J Neurol Neurosurg Psychiatry 76(7):965-970

10. Weerakkody RA, Czosnyka M, Schuhmann MU, Schmidt E, Keong N, Santarius T, Pickard JD, Czosnyka Z (2011) Clinical assessment of cerebrospinal fluid dynamics in hydrocephalus. Guide to interpretation based on observational study. Acta Neurol Scand 124(2):85-98 